\title{
DESIGN OF 3D TOPOLOGICAL DATA STRUCTURE FOR 3D CADASTRE OBJECTS
}

\author{
Nur Amalina Zulkifli*, Alias Abdul Rahman and Muhammad Imzan Hassan \\ Department of Geoinformation, Faculty of Geoinformation and Real Estate, Universiti Teknologi Malaysia, UTM \\ Skudai 81310, Johor, Malaysia - (amalina.jc@gmail.com, alias@utm.my and imzan@utm.my)
}

KEYWORDS: 3D topology, data structure, TEN, 3D cadastre, modelling

\begin{abstract}
:
This paper describes the design of 3D modelling and topological data structure for cadastre objects based on Land Administration Domain Model (LADM) specifications. Tetrahedral Network (TEN) is selected as a 3D topological data structure for this project. Data modelling is based on the LADM standard and it is used five classes (i.e. point, boundary face string, boundary face, tetrahedron and spatial unit). This research aims to enhance the current cadastral system by incorporating 3D topology model based on LADM standard.
\end{abstract}

\section{INTRODUCTION}

Traditionally, cadastral registrations consisted of a set of cadastral maps containing cadastral land parcels with unique parcel numbers and a paper archive in which property information on land parcels was maintained. Cadastral modelling should based on international standard specifications (i.e. ISO 19152 - LADM). The Land Administration Domain Model (LADM) is not to replace the existing system but rather to provide a formal language. According to Lemmen (2012), the reason behind LADM is to reuse the collective knowledge of many countries in land administration and to have clear definitions of the key concepts.

Cadastre has played a huge role in the development of our society since it aims at registering legal status and property rights associated with land. Cadastre is usually based on a $2 \mathrm{D}$ parcel description with associated land information. A number of countries have considered the adoption of the LADM to their local needs, especially in cadastral applications. For example, documentation in ISO 19152 (2012) including country profiles for Portugal, Australia, Indonesia, Japan, Hungary, the Netherlands, the Russian Federation and the Republic of Korea. Elia et al., (2013) investigated the adaptation of LADM in the Cyprus Land Information System (CLIS) with the aim of improving its data model. In Portugal, an object-oriented conceptual model based on LADM has been developed for the Portuguese Cadastre and the Portuguese Real Estate Register (Hespanha et al., 2008). Pouliot et al., (2013) used the LADM in comparative case study between condominium and coownership in Quebec (in Canada) and Alsace Moselle (in France). Most of the literature only propose the conceptual model for their country and rarely involve with implementation stage in order to validate the model.

Cadastral registrations throughout the world are based on the principle that a land parcel is the basic registration entity for cadastral registration. This principle of cadastral registration follows the juridical definition of ownership of land, which says that ownership of land is defined by boundaries on the surface and is not explicitly limited in the vertical dimension. In general, the ownership of land includes all space above and below land parcel, as well as all constructions that are permanently fixed to the land.

Pressure on land in urban areas has led to overlapping constructions. Describing the overlapping property rights in the cadastral registration pose a challenge. The challenge is how to register overlapping constructions in cadastral registration that registers information on $2 \mathrm{D}$ land parcels. Therefore, the cadastral registration should be extended into the third dimension. The basic needs for a 3D cadastre are to have a complete registration of $3 \mathrm{D}$ rights and to have good accessibility to the legal status of stratified property including 3D spatial information as well as to public law restrictions.

2D cadastral systems of most countries have been established and applied in years. To completely replace current 2D cadastral system by 3D cadastral system is cost expensive for the government. And constructing a full 3D cadastral database is an abandon of current cadastral data. It is important to utilize current cadastral data in 3D cadastre developments (Aien et al., 2011). The ISO 19152 (LADM) has been designed to allow parcel and boundary for $2 \mathrm{D}$ and $3 \mathrm{D}$ to be integrated together (Lemmen et al., 2010). However, to construct the 3D cadastral system and database based on current systems and data in practice is key issue need to be explored. Constructing of 3D data models and their topological relations are two important parts of 3D cadastral and its should based on international standard specifications (LADM - ISO 19152).

The use of topology in the cadastral application can be grouped into two areas. The first is to support spatial analysis. This includes the connectivity between cadastral objects for neighbourhood analysis. The second use of topology is to support the development of the database. It can be used to discover structural problem with the database feature. For example, polygons that are not closed or overlapping between the cadastral objects. It can also be used to automate feature creation and ensure feature integration. The research on 3D topology in cadastre applications deserves further exploration for a better cadastral system in the future. Any implementation of topology for 3D cadastral should take into account the requirements of the LADM standard model, to support the visualization to be built in conjunction with the 3D topology. 
This paper is organized as follows. Section 2 discusses the needs and design of 3D topology data structure (i.e. TEN) based on LADM. Conclusion and future work are given in Section 3.

\section{THE NEEDS AND DESIGN OF 3D TOPOLOGICAL DATA STRUCTURE}

Nowadays, 3D spatial analysis is widely used in most applications include urban planning, preservation of historical sites and buildings, and even complex procedures in disaster management. Soon, users will move towards more complex spatial queries than existing 2D/3D queries. It is impractical for 3D spatial analysis if the processing spatial 3D objects without their topological relationships. To optimize storage of the information relating to the $3 \mathrm{D}$ geometric topology objects, a suitable structure is needed since $3 \mathrm{D}$ applications are increasingly used. A new data structure with topology seems useful for the application of 3D cadastre, where the topological issues are crucial.

Topological structures are generally used to represent planar or space partitions without redundancy. In planar partitions (i.e. 2D topological structures) and space partitions (i.e. 3D topological structures), spatial objects are defined on the basis of non-overlapping objects. A large number of 2D topological structures are already available in the literature. Many 3D topological structures are also reported but only a few of them have been tested for large data sets. A topological structure has many advantages in cadastral application:

- It avoids redundant storage

- It is easier to maintain the consistency of the data after editing

- It is more efficient during the visualization because less data have to be read from disk

- It is the natural data model for certain applications; e.g. during surveying, an edge is collected together with attributes to a boundary

- It is more efficient for certain query operations (e.g. find neighbors)

2D topological data structures are well established. However, research works on 2D topology based on LADM standard are very few. A number of 2D topological data structures have been introduced to design 2D topology model. Example of such data structures are Wing Edge, POLYVRT, Topologically Integrated Geographic Encoding and Referencing - TIGER, Triangular Irregular Network -TIN and Geographic Base File/Dual Independent Map Encoding - GBF/DIME. Most of the 2D topological structures are based on the planar graph theory. A planar graph can be represented on a plane with nodes at each intersection between edges. Each given oriented edge has one face on the right, and one face on the left (De la Lossa and Cervella, 1999).

Several types of 3D topological structures (i.e. Tetrahedral Network (TEN), 3D Formal Data Structure (3D FDS), Simplified Data Schema (SSS) and Radius Topology) have been introduced. 2D topology is well investigated while the research on $3 \mathrm{D}$ topology between volumetric geometries is still indistinct. Furthermore, the topology of 3D spatial objects is more difficult to analyze than $2 \mathrm{D}$ topology due to the complexity of 3D geometry. There are even more complex relationships exist between the primitives in 3D. In addition, the orientation is more difficult. The orientation of an outer ring of a face cannot simply be defined as counterclockwise anymore, but the orientation is now related to the volume the face is bounded to. Each face has two orientations including negative and positive and an edge could be within the boundary of more than 2 faces. The real 3D volumetric or solid objects should be constructed first with the input faces based on the standard model and storing the reference in the 3D topological model (refer Figure 1).

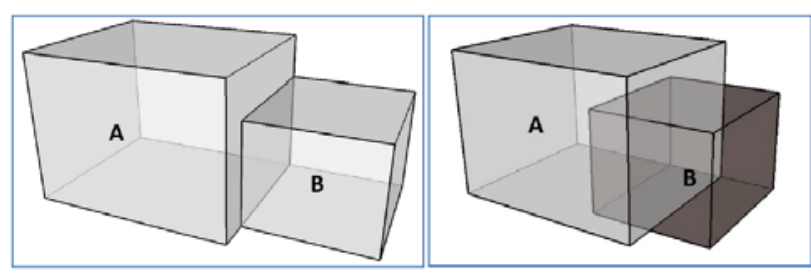

Figure 1. Correct topological relation (left) and incorrect topological relation (right) (after Zhao et al., 2012)

The identification of topological relations needs a topological data structure as the basis. Based on the reviews of the 3D topological data structures, the most suitable 3D topology data structure is a topological approach based on a tetrahedral network (TEN), proposed by (Penninga and van Oosterom, 2008). The TEN was selected as a structure due to its favourable characteristics from a computational point of view (i.e. only have four points). All elements of the tetrahedral network consist of flat faces, convex and they are all well defined because the three points of each triangle always lie in the same plane. In this paper, the topological data structure is based on TEN data structure. The data structure has been improved to fit the LADM standard specifications.

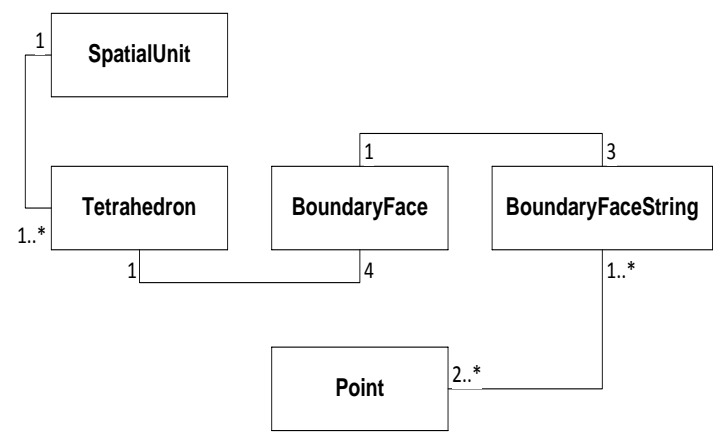

Figure 2. Conceptual model of TEN data structure based on LADM

The basic elements in a topological structure are nodes, edges and faces. A node is represented by a point. Every node has a coordinate pair associated with it to describe the location of the node. An edge is bounded by a start node and an end node and has a coordinate string describing the geometric representation. Each edge can consist of multiple vertices, represented by linear strings. As each edge is directed, it is possible to determine which faces are located on the left and right-hand side of the edge. A face corresponds to a polygon that can be reconstructed from several edge strings and has references to a directed edge on its outer and inner boundaries if any.

There are four elements are used in TEN data structure (i.e node, edge, face and tetrahedron). In LADM, the terms use for node, edge and face are different. According to LADM 
standard, nodes are stored in Point class, edges are stored in Boundary Face String class and faces are stored in Boundary Face class (refer Figure 2). The point is defined as 0demensional geometric primitive for position representation. Boundary Face String is a boundary forming part of the outside of a spatial unit. Meanwhile, a face that used in the representation of a boundary of a spatial unit is called Boundary Face. The spatial unit is a single area of land.

\section{CONCLUSION AND FUTURE WORK}

This paper shows the 3D modelling for cadastral application and some initial result of 3D topological data structure (i.e. TEN) based on LADM. Further investigation on this research is needed to come out with a complete 3D topological situation for 3D cadastral application. Nowadays, 2D cadastre is not capable of modeling the complex urban land use including underground structures and multi-storey building. The 2D land parcels cannot accommodate the increasing number of non-parcel interests. Many other problems for example overlapping and gap are also associated with the 2D cadastral model. To solve all these problems, a full 3D cadastral model based on LADM with a topology is needed to ensure that no overlaps or gaps in spaces.

\section{REFERENCES}

Aien, A., Rajabifard, A., Kalantari, M. and Williamson, I. (2011). Aspects of 3D Cadastre - A Case Study in Victoria. In proceedings FIG Working Week 2011, Marrakech, 15 p.

De la Losa, A. and B. Cervelle (1999). 3D topological modelling and visualisation for 3D GIS. In: computers and graphics.

Elia E, Zevenbergen J, Lemmen C, Van Oosterom P (2013). The land administration domain model (LADM) as the reference model for the Cyprus land information system (CLIS). Surv Rev. 45:100-10.

Hespanha J, van Bennekom-Minnema J, Van Oosterom P, Lemmen C. (2008). The model driven architecture approach applied to the land administration domain model version 1.1with focus on constraints specified in the object constraint language. In: fig working week. p. 19.

ISO (2012). ISO 19152: 2012 Geographic Information - Land Administration Domain Model (LADM), Version 1 December 2012.

Lemmen, C.H.J. (2012). A Domain Model for Land Administration. Ph.D. Thesis. Delft University of Technology, Delft, the Netherland.

Penninga, F. and van Oosterom, P.J.M. (2008). A Simplicial Complex-Based DBMS Approach to 3D Topographic Data Modelling, International Journal of Geographic Information Science, 22, 751-779.

Pouliot, J., Vasseur, M., Boubehrezh, A., (2013). How the ISO 19152 Land Administration Domain Model performs in the comparison of cadastral systems: A Case study of condominium/co-ownership in Quebec (Canada) and Alsace Moselle (France)", Special issue in Computers, Environment and Urban Systems, 40, p.68-78.
Zhao, Z., Guo, R., Li, L. And Ying S. (2012). Topological Relationship Identification in $3 D$ Cadastre. In proceedings of the $3^{\text {rd }}$ International Workshop on 3D Cadastres, 25-26 October 2012, Shenzhen, China, pp.345-360. 